\title{
Effect of Medical Advice for Diet on Diabetes Self-Management and Glycemic Control for Haitian and African Americans with Type 2 Diabetes
}

\author{
Fatma G. Huffman, Joel C. Exebio, Joan A. Vaccaro, Gustavo G. Zarini, Zisca Dixon
}

Department of Dietetics and Nutrition, Florida International University, Miami, USA.

Email: huffmanf@fiu.edu

Received July 22 $2^{\text {nd }}, 2013$; revised August 22 $2^{\text {nd }}, 2013$; accepted August $30^{\text {th }}, 2013$

Copyright (C) 2013 Fatma G. Huffman et al. This is an open access article distributed under the Creative Commons Attribution License, which permits unrestricted use, distribution, and reproduction in any medium, provided the original work is properly cited.

\begin{abstract}
Adequate care of type 2 diabetes is reflected by the individual's adherence to dietary guidance; yet, few patients are engaged in diabetes self-care at the recommended level, regardless of race/ethnicity. Few studies on the effect of dietary medical advice on diabetes self-management (DSM) and glycemic control have been conducted on Haitian and African American adults with type 2 diabetes. These relationships were assessed in total of 254 Blacks with type 2 diabetes (Haitian Americans = 129; African Americans = 125) recruited from Miami-Dade and Broward Counties, Florida by community outreach methods. Although dietary advice received was not significantly different between the two Black ethnicities, given advice "to follow a diet" as a predictor of "using food groups" was significant for Haitian Americans, but not for African Americans. Haitian Americans who were advised to follow a diet were approximately 3 times more likely to sometimes or often use food groups (or exchange lists) in planning meals. Less than optimal glycemic control (A1C > 7.2) was inversely related to DSM for African Americans; but the relationship was not significant for Haitian Americans. A one unit increase in DSM score decreased the odds ratio point estimate of having less than optimal glycemic control (A1C $>7.2 \%$ ) by a factor of 0.94 in African Americans. These results suggest that medical advice for diet plans may not be communicated effectively for DSM for some races/ethnicities. Research aimed at uncovering the enablers and barriers of diet management specific to Black ethnicities with type 2 diabetes is recommended.
\end{abstract}

Keywords: Dietary Medical Advice; Diabetes Self-Management; African American; Haitian American; Type 2 Diabetes; Glycemic Control

\section{Introduction}

Type 2 diabetes, the most common form (90\% - 95\% of all cases) has increased among the general population [1] and disproportionately among minorities (particularly African-Americans and Hispanics) [2]. Due to the many health consequences of diabetes and the nature of the disease, diabetes care is vital to quality of life and survival. It is essential for persons with diabetes to acquire and practice adequate diabetes self-management (DSM) skills in order to reduce the risk factors that lead to morbidity and mortality associated with diabetes-related complications [3]. DSM includes achieving adequate glycemic control, blood lipids and blood pressure as well as weight management through diet and exercise $[3,4]$. Nwasuruba, Khan and Egede [5] reported few patients are engaged in diabetes self-care at the recommended level, regardless of race/ethnicity using a US representative sample from the Behavioral Risk Factor Surveillance Survey (BRFSS). Despite the standards of care followed by health care professionals in treating persons with diabetes, nearly half of US adults with diabetes have suboptimal glycemic control (A1C $\geq 7 \%$ ) [6]. Minorities tend to have less access to and receive a lower quality of health care, even when controlling for insurance status and income [7]. Even when adjusting for socioeconomic status, the effects of race and/or ethnicity predict poor health outcomes (such as micro- and macro-vascular complications) due to a lack of cultural competency and appropriate communication skills by health care providers [7]. It is essential for persons with diabetes to acquire and practice adequate DSM skills in order to reduce the risk factors that lead to morbidity and mortality associated with 
diabetes-related complications. According to the Standard of Care for diabetes by the American Diabetes Association, dietary modification, weight management and incorporation of physical activity into the lifestyle of persons with diabetes are essential components of DSM [3]. Coping with diabetes has been widely associated with diagnosed depression [8] (and references therein). Depressive symptoms were associated with higher glycated hemoglobin (A1C) for African Americans (AA) with diabetes [9]. A recent study found Haitian Americans (HA) were more likely to have A1C $>9 \%$ as compared to AA, even when adjusting for socio-demographics [10]. The association of medical advice with DSM and glycemic control may be confounded by depression and perceived stress. Moreover, DSM may be a consequence of access to quality medical care and medical advice received and may differ between these Black ethnicities. There have been few studies on the effect of dietary advice on DSM and glycemic control for Haitian and African American adults (HA and AA) with type 2 diabetes. Therefore, the purpose of this study was to assess these relationships while adjusting for socio-demographic factors and coping abilities measured by depresssive symptoms and perceived stress. It is hypothesized for both HA and AA that 1) medical advice for following a meal plan will be associated currently following the advice; and, 2) high overall diabetes self-management will be associated with adequate glycemic control (lower A1C). For details of hypothesis variable construction and measurements, refer to the Measures section of Methods.

\section{Methods}

\subsection{Participants}

The study participants were recruited by community outreach methods from a larger study of HA and AA with and without diabetes, 2007-2010. Recruitment of the participants was from Miami-Dade and Broward Counties, Florida by community outreach methods. Letters of invitation outlining the study were mailed to diabetes educators and health professionals, requesting their cooperation in recruiting individuals with type 2 diabetes. Invitational flyers were distributed to all Florida International University (FIU) faculty, staff and students explaining the research protocol and requesting their assistance in the study. Advertisements were placed in local AA and HA newspapers and principal gathering places along with radio ads on local Creole and African American stations. In addition to community outreach, African Americans were mailed invitational flyers from a purchased mailing list from Knowledge Base Marketing, Inc., Richardson, TX, US. Inclusion criteria consisted of: male and female HA and AA adults with self-reported type 2 diabetes, age $\geq 35$ years, not taking insulin, not pregnant or lactating, no thyroid disorders, no coronary heart disease, not undergoing chemo or radiation therapy, not diagnosed with cancer, HIV, or AIDS, and no major psychiatric disorders. Respondents with diabetes were asked for the age they were diagnosed and their initial treatment modalities (with or without insulin). Inquiry of ethnicity included questions of cultural identification and place of birth. Diabetes status (diagnosed with type 2 diabetes or not) was determined by reported year of diagnosis and then confirmed by laboratory reports. Individuals with fasted blood glucose $\geq 126 \mathrm{mg} / \mathrm{dL}$ were classified as having diabetes. Eligibility was determined by interviewers' screening, and respondents' self-report. Those participants undiagnosed, but considered having diabetes based on their laboratory reports, were not included in this study; however, they were given their laboratory reports and advised to contact their physician. A total of 254 participants with type 2 diabetes were included in this study $(\mathrm{HA}=129$; $\mathrm{AA}=125)$. The study was approved by the Institutional Review Board of Florida International University. All participants read, understood and signed an informed consent form in either English or Creole.

\subsection{Measures}

Main study variables: Medical advice given to follow a meal plan or diet, level of reporting following a diet, and the diabetes self-management (DSM) composite score was collected as part of the Michigan Diabetes Questionnaire [11]. Dietary advice for individuals with type 2 diabetes suggests using exchange lists or food groups in meal planning [12]. This strategy is referred to as the "diabetes diet" and includes an individually tailored prescription to either use the exchange list for macronutrients, counting carbohydrates or use the glycemic index for meal plans [12].

Hypothesis 1, independent variable: The following question from the section on Educational/Advice Received was used: "Has your health care provider or nurse ever told you to follow a meal plan or diet?" The possible responses were yes, no, or not sure. Since all participants answered yes or no, the variable was measured as binary.

Hypothesis 1, dependent variable: "How often do you (or the person who cooks your food) use the exchange lists or food group lists to plan your meals," answered on a 5-point scale (never, rarely, sometimes, often, always) used as dependent variable for hypothesis 1 . Based on the estimated marginal means, the variable was collapsed to a binary variable. Sometimes, often, and always were considered "using food groups to plan meals" versus rarely and never. 
Hypothesis 2, independent variable: The diabetes selfmanagement (DSM) score was constructed as composite scales based on the major subscales from the Michigan Diabetes Research and Training Center (MDRTC) Diabetes Care Profile questionnaire [11]. This questionnaire has been widely used and was validated and tested for reliability for Caucasians and African Americans by several studies conducted by Fitzgerald and colleagues [11]. The composite score for DSM was constructed from the Likert sub-scale variables. Variables where higher scores indicated clinically appropriate DSM were added directly to the composite score. Exercise barriers were reversecoded so that rarely having trouble getting exercise responses reflected a higher DSM. The following subscales were combined to form the DSM composite score: 1) DSM care adherence (I keep my blood sugar in good control; I keep my glycated hemoglobin (A1C) in good control; I keep my weight under control; I do the things I need to do for my diabetes (diet, medicine, exercise, etc.); 2) dietary patterns (following a meal plan; scheduling meals and snacks; weighing or measuring food; meal planning (by you or the person who cooks) such as exchange list or food groups); 3) exercise barrier scale: How often do you have trouble getting enough exercise because: it takes too much effort? You don't believe it is useful? You don't like to do it? You have a health problem? It makes diabetes more difficult to control? and, 4) health beliefs: Taking the best possible care of diabetes will delay or prevent: 1 . eye problems; 2. kidney problems; 3. foot problems; 4. hardening of the arteries; 5. heart disease. The DSM composite scale score followed a normal distribution. Reliability was measured for the sub-scales using Cronbach's alpha. Cronbach's alpha value of 0.70 to 0.79 is considered to be acceptable, 0.80 to 0.89 to be very good and 0.90 and higher to be excellent [13]. All items fell in the "very good" range except the DSM-exercise subscale, which was considered marginal (alpha $=0.721)$; removing an item did not raise the value (Table 1).

Hypothesis 2, dependent variable: Sub-optimal glycemic control was considered A1C $>7.2$. This cutoff was applied using the median and in consideration of the American Diabetes Association's guideline [3] for setting goals for glycemic control as A1C either below 7 or

Table 1. Reliability of the Diabetes self-management composite scale.

\begin{tabular}{ccc}
\hline Sub-scale & Number of items & Crombach's alpha \\
\hline DSM care adherence & 4 & 0.813 \\
DSM dietary patterns & 4 & 0.800 \\
Exercise for DSM & 5 & 0.721 \\
DSM health beliefs & 5 & 0.854 \\
\hline
\end{tabular}

Abbreviation: DSM = diabetes self-management.
8 percent depending on the characteristics of the patient. A1C was measured from a $20 \mathrm{ml}$ sample of venous blood collected from each participant after an overnight fast $(8$ - $10 \mathrm{~h}$ ) by a certified phlebotomist using standard laboratory techniques. Whole-blood samples for $\mathrm{A} 1 \mathrm{C}$ were collected in a tube containing EDTA and A1C percentages were measured using Roche Tina Quant method by the Laboratory Corporation of America (LabCorp ${ }^{\circledR}$, Miami, FL, USA).

\subsection{Covariates}

Sociodemographics: Age, gender, duration of residence in the USA, language preference, education, income, employment status, medication history, family history of diabetes and coronary heart disease were collected using a socio-demographic questionnaire.

Psychosocial variables: Perceived stress, self-rated health, and symptoms of depression were assessed, since they could be confounders of health behavior. To measure perceived stress, the Perceived Stress Scale (PSS-10) $[14,15]$ was administered with permission from the John E. and Catherine MacArthur Research Network on Socioeconomic Status and Health and was scored and constructed as a continuous variable based on the author's guidelines. The sociodemographic questionnaire included self-rated health (SRH) which was measured in response to the question, "In general, would you say your health is: (Check one box)." Self-rated health was measured on a 5-point scale (excellent, very good, good, fair and poor). SRH was collapsed to a binary variable (fair/ poor versus good, very good, excellent) based on the distribution of participants.

Depressive symptoms: The questionnaire used was the Beck Depression Inventory (BDI). The BDI is a 21-item, self-reported questionnaire that measures the presence and severity of depressive symptoms using a self-rating scale from 0 to 3 ( 0 being least depressed and 3 being most depressed). The scale has been previously validated against Caucasians in Hispanic [16] and Black populations [17]. Scores for each question were summed, calculating a final BDI score.

Anthropometrics: Participant's height and weight were measured with the subject standing erect without shoes, and for weight, wearing light clothes using a SECA clinical scale (SECA Corp, Columbia, MD). Height and weight were measured to the nearest $0.5 \mathrm{~cm}$ and $0.1 \mathrm{~kg}$, respectively. Body mass index (BMI) was calculated as weight $(\mathrm{kg})$ divided by height $(\mathrm{m})$ squared $\left(\mathrm{kg} / \mathrm{m}^{2}\right)$.

Physical activity was estimated from the Modifiable Activity Questionnaire (MAQ) [18]. The MAQ was used to estimate metabolic cost and perform a metabolic equivalence (MET) calculation, which is explained in 
detail in the compendium of physical activity [19]. Physical activity was expressed as metabolic equivalents per hour per week $\left(1 \mathrm{kcal} \cdot \mathrm{kg}^{-1} \cdot \mathrm{h}^{-1}\right)$. One MET is the equivalent of sitting quietly. The participants, with the help of a trained interviewer, estimated the number of months and frequency per month and duration of each activity. For occupational activity, participants reported all jobs held over the past year. The number of hours sitting at work and usual mode of transportation to work was recorded for each job. Total physical activity was calculated, summing all leisure and occupational activity hours per week. These values were then multiplied by their estimated metabolic cost.

\subsection{Statistical Analysis}

Demographic characteristics, medical advice and diabetes self management scores were compared among HA and AA using the Student $t$-test for continuous variables and Chi-Square test for categorical variables. Logistic regression models were used to test hypothesis 1 , where "advised to follow a diet" (yes/no) was the major independent variable and "using exchange lists or food groups to plan meals" (never/seldom versus sometimes/ often/always) was the binary outcome. Since there was an interaction between ethnicity and medical advice for diet, separate analyses were conducted for HA and AA. Both unadjusted and adjusted models were conducted. Two and three way interaction terms between told to follow a diet, gender and education level were tested. Covariates included in the model were diabetes medication, stress score, age, gender, BMI, years with diabetes, self-rated health, health insurance, depression, A1C, marital status, education, smoking, and physical activity.

To test hypothesis 2 , logistic regression models were used where diabetes self management score (continuous) was the main independent and a binary measure of suboptimal glycemic control $(\mathrm{A} 1 \mathrm{C}>7.2)$ was constructed as the outcome, based on the sample distribution and the American Diabetes Association's glycemic goals for adults [3]. Two and three way interaction terms between diabetes self management score, race, gender and education level were tested. Statistical analysis was conducted using SPSS version 18.0 and significance was set at $P<0.05$.

\section{Results}

Characteristics of the study population are presented in Table 2. Although sociodemographic factors differed between HA and AA, the main study variables: advised to follow a diet, level of following a diet (using food

Table 2. General characteristics of the study population.

\begin{tabular}{|c|c|c|c|}
\hline Variable & Haitian-Americans $(n=129)$ & African-Americans $(n=125)$ & $P$-value \\
\hline Age (years) & $58.0 \pm 9.9$ & $54.2 \pm 10.1$ & 0.002 \\
\hline Gender (female) & $55.8(72)$ & $52.0(65)$ & 0.615 \\
\hline BMI $\left(\mathrm{kg} / \mathrm{m}^{2}\right)$ & $29.4 \pm 5.3$ & $35.6 \pm 8.6$ & $<0.001$ \\
\hline Told to follow a diet (yes) & $72.1(93)$ & $77.6(97)$ & 0.386 \\
\hline Using food groups (sometimes or often) & $11.0 \pm 7.8$ & $9.7 \pm 9.7$ & 0.262 \\
\hline Poor glycemic control (A1C > 9\%) & $27.9(36)$ & $20.8(26)$ & 0.193 \\
\hline DSM & $68.2 \pm 9.3$ & $63.4 \pm 10.2$ & $<0.001$ \\
\hline Duration of diabetes (years) & $9.4 \pm 7.4$ & $9.1 \pm 8.7$ & 0.721 \\
\hline Diabetes medication (yes) & $89.9(116)$ & $76.8(96)$ & 0.006 \\
\hline SRH (fair or poor) & $51.9(67)$ & $45.6(57)$ & 0.132 \\
\hline Health insurance (yes) & $55.0(71)$ & $79.2(99)$ & 0.001 \\
\hline Physical activity (MET hours/week) & $34.7 \pm 60.1$ & $39.1 \pm 67.0$ & 0.576 \\
\hline Marital status (married) & $62.0(80)$ & $26.4(33)$ & $<0.001$ \\
\hline Education (high school or higher) & $45.0(58)$ & $82.4(103)$ & $<0.001$ \\
\hline Currently smoking (yes) & $6.9(9)$ & $34.4(43)$ & $<0.001$ \\
\hline BDI Depression score & $55.8(72)$ & $47.2(59)$ & 0.211 \\
\hline Perceived Stress score & $17.2 \pm 7.3$ & $15.7 \pm 8.4$ & 0.171 \\
\hline
\end{tabular}

Abbreviations: $\mathrm{BMI}=$ body mass index; $\mathrm{A} 1 \mathrm{C}=$ percent of glycated hemoglobin; $\mathrm{BDI}=$ beck depression inventory; $\mathrm{DSM}=$ diabetes self-management; $\mathrm{SRH}=$ self-rated health; MET $=$ metabolic equivalents (per hour per week). MET $=\left(1 \mathrm{kcal} \cdot \mathrm{kg}^{-1} \cdot \mathrm{h}^{-1}\right)$. One MET is the equivalent of sitting quietly. Notes: Data are reported as $\mathrm{N}(\%)$ for categorical variables and mean \pm standard deviation (SD) for continuous variables. $P<0.05$ is considered significant. 
groups to plan meals), and glycemic control were not significantly different between the Black ethnicities; albeit DSM was higher for HA than AA $(P<0.001)$. A number of possible confounders of health behavior: duration of diabetes, SRH, depression symptoms, physical activity, and perceived stress were not significantly different between HA and AA. Several risk factors were more prevalent in AA participants than HA: obesity, as measured by BMI, and smoking; whereas HA had a lower percent of health care insurance.

Hypothesis 1: Dietary advice and behavior was not independent of race/ethnicity. The combined model race/ ethnicity by "given advice to follow a diet" was not significant, so separate logistic regression analyses were conducted for HA and AA. "Given advice to follow a diet" as a predictor of "using food groups" was significant for HA, but not for AA. HA who were advised to follow a diet were approximately 3 times more likely to sometimes or often use food groups (or exchange lists) in planning meals in both the unadjusted and adjusted models (Table 3). Effects of the covariates: No covariates were significant for dietary advice and behavior.

Hypothesis 2: DSM and glycemic control were also not independent of race/ethnicity. Suboptimal glycemic control (A1C > 7.2) was inversely related to DSM for AA in unadjusted and adjusted models; whereas this relationship was not significant for HA. A one unit increase in DSM score decreased the odds ratio point. Estimate of having suboptimal glycemic control (A1C > 7.2) by a factor of 0.94 in AA (Table 3).

Effects of the covariates: Of the adjustment variables for DSM and glycemic control, only BMI $(P=0.026)$, years with diabetes $(P=0.004)$, and physical activity $(P$ $=0.028)$ were significant.

\section{Discussion}

In the current study, dietary advice was associated with performing the behavior for HA, but not for AA; whereas, overall DSM skills, beliefs, and behaviors were associated with glycemic control for AA, but not HA. This finding may be explained, in part, by the high percent of HA participants without health insurance $(45 \%)$ as compared to AA (20\%). Although diabetes care is largely the responsibility of the individual, healthcare providers play a vital role in the patient's skill development. In fact, health care providers are the link between the patient and their disease self-management. The communication process between the provider and patient can determine whether or not the patient is informed, motivated and confident enough to make the behavioral changes necessary for diabetes care. Federal agencies and the literature concur that access to quality health care and education for diabetes self-management are essential to the prevention of diabetes complications. Quality health care can be assessed by the degree to which diabetes complications are prevented or reduced as a result of patient's lifestyle behavioral changes. Medical advice associated with diabetes self-management and clinical health outcomes can serve as measures of risk for diabetes complications for a cross-sectional sample. The association among provider support for diabetes self-management, patient self-care and health outcomes is presumed, yet understudied [20]. In this sample of AA, DSM may serve as a proxy measure of glycemic control. A low DSM score may be used as a screening tool for tighter glycemic control confirmed later with $\mathrm{A} 1 \mathrm{C}$ testing.

Heisler, Cole, Weir, Kerr and Hayward [21] using two validated scales to access medical advice found that when providers communicated information and allowed

Table 3. Logistic regression models for the effect of dietary advice and diabetes self-management on dietary behavior and glycemic control.

\begin{tabular}{|c|c|c|c|c|c|c|}
\hline & OR & $95 \% \mathrm{CI}$ & $P$-value ${ }^{\dagger}$ & $\mathrm{AOR}^{*}$ & $95 \% \mathrm{CI}$ & $P$-value ${ }^{\dagger}$ \\
\hline Model 1 Dietary advice & \multicolumn{6}{|c|}{$\begin{array}{l}\text { Odds ratio of "using an exchange list or food groups to plan meals" always/most } \\
\text { of the time/sometimes versus seldom/never }\end{array}$} \\
\hline Given advice to follow a diet & \multicolumn{6}{|c|}{ Haitian Americans } \\
\hline Yes $(n=93)$ & 2.98 & $(1.34,6.63)$ & 0.007 & 3.27 & $(1.32,8.00)$ & 0.010 \\
\hline No $(n=36)$ & 1.00 & Reference & & 1.00 & Reference & \\
\hline \multirow[t]{3}{*}{ Model 2 Diabetes self-management } & \multicolumn{6}{|c|}{ African Americans } \\
\hline & \multicolumn{6}{|c|}{ DSM score and odds ratio point estimate of having suboptimal glycemic control (A1C > 7.2) } \\
\hline & OR & $95 \% \mathrm{CI}$ & $P$-value ${ }^{\dagger}$ & $\mathrm{AOR}^{*}$ & $95 \%$ CI & $P$-value ${ }^{\dagger}$ \\
\hline DSM score & 0.94 & $(0.90,0.97)$ & 0.002 & 0.92 & $(0.87,0.98)$ & 0.009 \\
\hline
\end{tabular}

$\mathrm{OR}=$ Odds Ratio; AOR = adjusted Odds Ratio; DSM = diabetes self-management; A1C = percent of glycated hemoglobin. ${ }^{*}$ Models 1 and 2 are adjusting for diabetes medications, perceived stress score, age, gender, body mass index, years with diabetes, self-rated health, health insurance, depression, marital status, education, smoking, and physical activity. In addition, Model 1 is adjusted for A1C. ${ }^{\dagger} P<0.05$ is considered significant. 
patients to be involved in decisions, diabetes self-management practices (medication adherence, diet, exercise, blood glucose monitoring and foot care) and $\mathrm{A} 1 \mathrm{C}$ values improved for a national cross-sectional survey of 1588 older adults (Blacks and Latinos were oversampled) with diabetes. Improvement in diet and $\mathrm{A} 1 \mathrm{C}$ was associated with a continuity of care score (number of visits to health care providers and the number of providers seen) for a prospective study of 256 adults ages 18 and older with type 2 diabetes [22]. A longitudinal study of four age groups of urban African-Americans with type 2 diabetes at a primary health care clinic $(\mathrm{N}=2539)$ reported a significant improvement of $\mathrm{A} 1 \mathrm{C}$ for those treated with dietary advice and diabetes medications in all age groups $(P$ $<0.001$ ); however, patients with less frequent visits were associated with higher A1C levels [23].

Medical advice received and understood may differ by race/ethnicity as a consequence of the patient-provider communication process; and, may affect diabetes selfmanagement. AA were less-likely to perform a composite of four diabetes self-management behaviors, including physical activity, diet, self-testing blood glucose and foot care, as compared to White non-Hispanics based on a nationally representative survey [5]. Similar racial/ethnic differences in diabetes self-management were found in a survey of persons with diabetes in a managed care setting: AA had lower odds of monitoring their diet and exercising as compared to White non-Hispanics [24].

The interpretation of medical advice received may be further complicated by the patient's health beliefs, which can serve as barriers to their self-care. Health beliefs, compliance and guidelines for spiritual assessment addressed by The National Medical Association and the Joint Commission on Accreditation of Healthcare Organizations are of particular importance for AfricanAmerican patients [25]. Health beliefs that contribute to non-adherence may differ by culture. For example, Haitian-Americans' high rate of noncompliance has been attributed, in part, to their health beliefs [26]. HaitianAmerican beliefs about health and illness may be influenced by life in Haiti where limited access to health care and poor health conditions influenced a reliance on folk and/or spiritual explanations and treatments for illness [26]. Yet, Haitians have a strong set of protective factors that are conducive to health educational programs including a strong work ethic; entrepreneurial spirit, extended family support system and increasing neighborhood-based social services [27].

Findings of health beliefs and compliance with African-Americans suggest multiple influences, including religion, spirituality and folklore [25]. African-Americans were found to be more than twice as likely to use home-remedies as Whites [28]. Several studies concur that factors beyond knowledge and access to medical care such as noncompliance and lay beliefs inconsistent with medical practice are responsible for inadequate hypertensive control among this population [29-31]. Nwasuruba, Khan, and Egede [5] found significant differences among Blacks, Hispanics and White non-Hispanics in DSM behaviors, based on data from the 2003 Behavioral Risk Factor Surveillance survey (BRFSS); however, data did not specify different origins of persons classified as "Black."

\subsection{Limitations}

Several limitations were present in this study. Medical advice and diabetes self-management behavior were self-reported. The study was cross-sectional and causality of medical recommendations and following the advice could not be established. The study represents a single time point and there are other environmental, social influences that combined with individual characteristics in determining behavior. For example, patient-provider communication and health beliefs were not measured. There is no absolute way of determining the level of medical advice that was actually given. For instance, medical advice may have been given and there could have been problems with communication or recall. In some cases, the medical advice was given and the patient did not remember receiving it at the time of the survey (lack of recall). In other cases the patient received the advice but did not process it (ineffective communication on the part of the physician or healthcare provider).

\subsection{Conclusion}

In the present study there were differences in medical advice for diet and following it for Blacks of two ethnicities. Healthcare providers should consider cultural background, coping abilities, and sociodemographic factors when providing guidance and education for patients to develop strategies to manage their diabetes.

\section{Acknowledgements}

This grant was funded by the National Institutes of Health: NIH/NIDDK \#1SC1DK083060-01 to the corresponding author.

\section{REFERENCES}

[1] National Institute of Diabetes and Digestive Kidney Diseases, "National Diabetes Statistics, 2011 Fact Sheet," US Department of Health and Human Services, National Institutes of Health, Bethesda, 2011.

[2] Center for Disease Control and Prevention (CDC), "National Diabetes Fact Sheet," 2011. 
http://www.cdc.gov/diabetes/pubs/pdf/ndfs_2011.pdf

[3] American Diabetes Association, "Standards of Medical Care in Diabetes-2012," Diabetes Care, Vol. 35, Suppl. 1, 2012, pp. S4-S63. http://dx.doi.org/10.2337/dc12-s004

[4] M. W. Stolar, B. J. Hoogwerf, S. M. Gorshow, P. J. Boyle and D. O. Wales, "Managing Type 2 Diabetes: Going beyond Glycemic Control," Journal of Managed Care Pharmacy, Vol. 14, No. 5, 2008, pp. S2-S19.

[5] C. Nwasuruba, M. Khan and E. Egede, "Racial/Ethnic Differences in Multiple Self-Care Behaviors in Adults with Diabetes," Journal of General Internal Medicine, Vol. 22, 2007, pp. 115-120. http://dx.doi.org/10.1007/s11606-007-0120-9

[6] Agency for Healthcare Research and Quality, "National Healthcare Quality Report, 2008. Chapter 2: Effectiveness, Diabetes," AHRQ Publication No. 09-000, 2008.

[7] American College of Physicians, American Society of Internal Medicine, "No Health Insurance? It's Enough to Make You Sick. Latino Community at Great Risk," White Paper, Philadelphia, ACP-ASIM, 2000.

[8] G. A. Nichols and J. B. Brown, "Unadjusted and Adjusted Prevalence of Diagnosed Depression in Type 2 Diabetes," Diabetes Care, Vol. 26, No. 3, 2003, pp. 744749. http://dx.doi.org/10.2337/diacare.26.3.744

[9] J. A. Wagner, G. L. Abbott, A. Heapy and L. Yong, "Depressive Symptoms and Diabetes Control in African Americans," Journal of Immigrant Minority Health, Vol. 11, No. 1, 2009, pp. 66-70. http://dx.doi.org/10.1007/s10903-008-9147-1

[10] V. G. Vimalananda, J. L. Rosenzweig, H. J. Cabral, M. M. David and K. E. Lasser, "Comparison of Diabetes Control among Haitians, African Americans, and Non-Hispanic Whites in an Urban Safety-Net Hospital," Diabetes Care, Vol. 34, No. 1, 2011, pp. 58-60. http://dx.doi.org/10.2337/dc10-1387

[11] Michigan Diabetes Research and Training Center, "Survey Instruments for Health Professionals: Diabetes Care Profile," 2012. http://www.med.umich.edu/mdrtc/profs/survey.html\#dcp

[12] American Diabetes Association, "Using Exchange Lists: Diabetes Meal Planning,” 2013.

http://www.americandiabetes.com/living-diabetes/diabete s-nutrition-articles/using-exchange-lists-diabetes-meal-pla nning

[13] J. Cohen, "Statistical Power Analysis for the Behavioral Sciences," 2nd Edition, Lawrence Erlbaum Associates, Hillsdale, 1988, p. 535.

[14] S. Cohen, T. Kamarck and R. Mermelstein, "A Global Measure of Perceived Stress," Journal of Health and Social Behavior, Vol. 24, No. 4, 1983, pp. 385-396. http://dx.doi.org/10.2307/2136404

[15] S. Cohen and G. Williamson, "Perceived Stress in a Probability Sample of the U.S.," In S. Spacapam and S. Oskamp, Eds., The Social Psychology of Health: Claremont Symposium on Applied Social Psychology, Sage, Newbury Park, 1988, pp. 99-125.

[16] S. Contreras, S. Fernandez, V. L. Malcarne, R. E. Ingram and V. R. Vaccarino, "Reliability and Validity of the Beck Depression and Anxiety Inventories in Caucasian Americans and Latinos," Hispanic Journal of Behavioral Sciences, Vol. 26 No. 4, 2004, pp. 446-462. http://dx.doi.org/10.1177/0739986304269164

[17] K. B. Grothe, G. R. Dutton, G. N. Jones, J. Bodenlos, M. Ancona and P. J. Brantley, "Validation of the Beck Depression Inventory-II in a Low-Income African American Sample of Medical Outpatients," Psychological Assessment, Vol. 17, No. 1, 2005, pp. 110-114. http://dx.doi.org/10.1037/1040-3590.17.1.110

[18] A. M. Kriska, W. C. Knowler, R. E. LaPorte, et al., "Development of Questionnaire to Examine Relationship of Physical Activity and Diabetes in Pima Indians," Diabetes Care, Vol. 13, No. 4, 1990, pp. 401-411. http://dx.doi.org/10.2337/diacare.13.4.401

[19] B. E. Ainsworth, W. L. Haskell, S. D. Herrmann, et al., "Compendium of Physical Activities: A Second Update of Codes and MET Values," Medicine and Science in Sports and Exercise, Vol. 43, No. 8, 2011, pp. 1575-1581. http://dx.doi.org/10.1249/MSS.0b013e31821ece12

[20] J. Greene and M. J. Yedidia, "Provider Behaviors Contributing to Patient Self Management of Chronic Illness among Underserved Populations," Journal of Health Care for the Poor and Underserved, Vol. 16, No. 4, 2005, pp. 808-824. http://dx.doi.org/10.1353/hpu.2005.0097

[21] M. Heisler, I. Cole, D. Weir, E. A. Kerr and R. A. Hayward, "Does a Physician Communication Influence Older Patients' Diabetes Self-Management and Glycemic Control? Results from the Health and Retirement Study," Journal of Gerontology, Vol. 62A, No. 12, 2007, pp. 1435-1442.

[22] M. L. Parchman, J. A. Pugh, P. H. Noel and A. C. Larme, "Continuity of Care, Self-Management Behaviors, and Glucose Control in Patients with Type 2 Diabetes," Medical Care, Vol. 40, No. 2, 2002, pp. 137-144. http://dx.doi.org/10.1097/00005650-200202000-00008

[23] I. M. El-Kebbi, C. B. Cook, D. C. Ziemer, C. D. Miller, D. L Gallina and L. S. Phillips, "Association of Younger Age with Poorer Glycemic Control and Obesity in Urban African Americans with Type 2 Diabetes," Archives of Internal Medicine, Vol. 163, No. 1, 2003, pp. 69-75. http://dx.doi.org/10.1001/archinte.163.1.69

[24] N. V. Oster, V. Welch, L. Schil, J. A. Gazmararian, K. Rask and C. Spettell, "Differences in Self-Management Behaviors and Use of Preventive Services among Diabetes Management Enrollees by Race and Ethnicity," Disease Management, Vol. 9, No. 3, 2006, pp. 167-175. http://dx.doi.org/10.1089/dis.2006.9.167

[25] J. Levin, L. M. Chatters and R. J. Taylor, "Religion, Health and Medicine in African Americans: Implications for Physicians," Journal of the National Medical Association, Vol. 97, No. 2, 2005, pp. 237-249.

[26] C. Kemp, "Haitians," 2006. https://bearspace.baylor.edu/Charles_Kemp/www/haitian _refugees.htm

[27] G. Metellus, S. St. Hilaire, L. Hermantin and S. Lacroix, "Risk and Protective Factors in Little-Haiti and the Hai- 
tian/Haitian-American Community in Miami Dade County,” Working paper Series: SL WPS 02, 2004.

[28] C. M. Brown and R. Segal, "The Effects of Health and Treatment Perceptions on the Use of Prescribed Medication and Home Remedies among African American and White Hypertensives," Social Science and Medicine, Vol. 43, No. 6, 1996, pp. 903-917. http://dx.doi.org/10.1016/0277-9536(95)00434-3

[29] R. Wilson, "Lay Beliefs about High Blood Pressure in a Low- to Middle-Income Urban African-American Community: An Opportunity for Improving Hypertension
Control," American Journal of Medicine, Vol. 112, No. 1, 2002, pp. 26-30. http://dx.doi.org/10.1016/S0002-9343(01)01049-X

[30] L. M. Lewis and G. Ogedegbe, "Understanding the Nature and Role of Spirituality in Relation to Medication Adherence: A Proposed Conceptual Model," Holistic Nursing Practice, Vol. 22, No. 5, 2008, pp. 261-271.

[31] J. L. Middleton, “A Proposed New Model of Hypertensive Treatment Behavior in African Americans," Journal of the National Medical Association, Vol. 101, No. 1, 2009, pp. 12-17. 